\title{
A case report of takotsubo cardiomyopathy
}

\author{
Khushbu Sharma, Dheeraj Nair, Jibran Monga, Pallavi Sehgal \\ From Junior Resident, Department of Emergency Medicine, Max Super Speciality Hospital Vaishali, Ghaziabad, Uttar Pradesh, India
}

\begin{abstract}
Takotsubo cardiomyopathy (TC) is defined as the weakening of the left ventricle. It occurs exclusively in women, and it accounts for $1-2 \%$ of all cases of suspected acute myocardial infarction, its true prevalence is unknown due to underdiagnosis. It may present with a similar clinical picture mimicking acute myocardial infarction. We report the case of a 53-year-old woman who came to the emergency department with complaints of recurrent vomiting and decreased oral intake. After sometimes, the patient started complaining of chest pain, for which electrocardiography was done, which showed ST-segment elevation. Coronary angiography was done, which showed normal coronary arteries. Afterward, a stressor precipitant was identified. The patient was diagnosed with TC. TC is a great mimic of acute myocardial infarction due to its close resemblance to myocardial infarction in terms of clinical presentation and clinical course. It should be considered as one of the important differentials in patients presenting with acute chest pain.
\end{abstract}

Key words: Myocardial infarction, Stress-induced, Takotsubo cardiomyopathy

$\mathrm{T}$ akotsubo cardiomyopathy (TC) occurs as the result of severe emotional or physical stress, such as a sudden drop in the blood pressure, sudden illness, surgery, severe pain, domestic violence, the loss of loved ones, intense fear, a serious accident, or a natural disaster such as an earthquake. That's why the condition is also called stress-induced-cardiomyopathy or broken heart syndrome [1]. The shape of the ventricle at the end of the systole resembled the Japanese fisherman's octopus pot "the takotsubo," from which it derives its original name. It is now recognized that TC stressor is not just acute emotional stress but also an acute intracranial event, including intracranial bleeding, head trauma, and ischemic stroke; acute medical illness, including sepsis and surgical procedure; overproduction of endogenous catecholamines (pheochromocytoma); and administration of exogenous catecholaminergic agents can be precipitating factors for the disease [2]. TC has been recognized by the American heart association as a unique form of reversible cardiomyopathy [3].

\section{CASE REPORT}

A 53-year-old female was brought to the emergency with complaints of recurrent vomiting and decreased oral intake for three to 4 days. The patient was a known case of type 2 diabetes mellitus, hypertension, and metastatic carcinoma colon (on ongoing chemotherapy).

\section{Access this article online}

Received - 06 September

Initial Review - 22 September 2020

Accepted - 15 October 2020

DOI: $10.32677 /$ IJCR.2020.v06.i10.013
On examination, the vitals were as follows: a pulse rate of 68/ min, temperature of 98 degrees $\mathrm{F}$, respiratory rate of $18 / \mathrm{min}$, and blood pressure was non-recordable. The tongue was dry, and the patient was lethargic. The patient was initially managed with fluid resuscitation but was later put on inotropic and vasopressor support.

After sometimes, the patient started complaining of the chest pain, for which electrocardiography (ECG) was done, which showed ST-segment elevation in lead V2 to V4 (Fig. 1). Troponin I was done and the levels were found elevated (Trop I $-4.73 \mathrm{ng} / \mathrm{ml}$ ). In view of the deteriorating condition, the patient was put on mechanical ventilation and shifted to the Cath Lab.

A 2D echocardiography was performed which showed akinetic apical septum, hypokinetic, mid anterior septum with a left ventricular ejection fraction 35\% (Fig. 2). Coronary angiography was done, which shows normal coronary arteries (Fig. 3). Later on, the patient was admitted to the coronary care unit. After 3 days, the patient was extubated. Later on, the patient was discharged in a stable condition and was advised to follow-up in the outpatient department.

\section{DISCUSSION}

TC is considered to be among stress-related cardiomyopathies, which occur during times of enhanced sympathetic tone and can be precipitated by excessive endogenous or exogenous catecholamine stimulation of the myocardium, a condition that is also seen in cases of intracranial hemorrhage, ischemic stroke, head trauma, pheochromocytoma, and in critically ill patients. In the majority of the cases, TC most commonly

Correspondence to: Dr. Khushbu Sharma, C-1501, Risabh Cloud 9, Sector 1, Vaishali, Ghaziabad, Uttar Pradesh, India. E-mal: khushbusharma082@gmail.com

(C) 2020 Creative Commons Attribution-NonCommercial 4.0 International License (CC BY-NC-ND 4.0). 


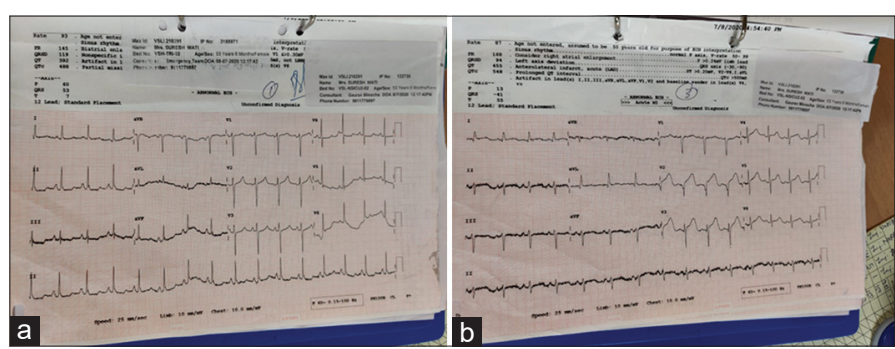

Figure 1: (a) Initial electrocardiography; (b) After complaining of the chest pain (ST elevation in lead V2 to V4)

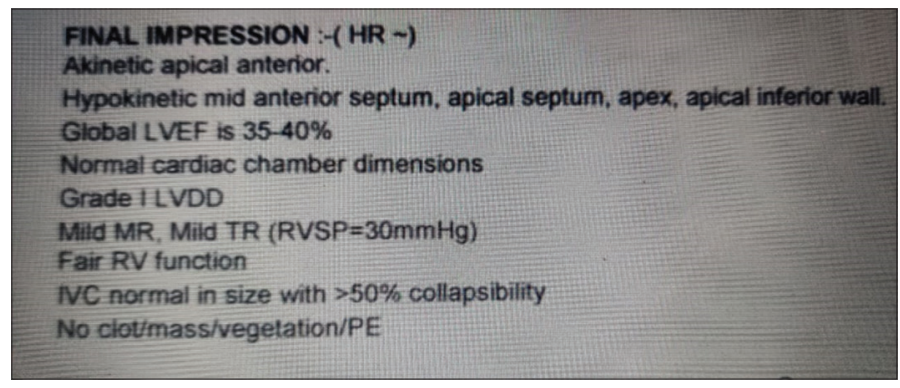

Figure 2: 2D echocardiography

affects postmenopausal women. In a systematic review, women accounted for $90 \%$ of patients with an average age of 53-75 years. Research suggests that up to $5 \%$ of women suspected of having an acute myocardial infarction actually have this disorder [2]. The first case reported in Mexico was in 2004 and then in 2011, two more cases were reported in just 1 year. Therefore, it is suggested that a lack of awareness about the disease is one of the reasons for underdiagnosis $[4,5]$.

Several mechanisms have been implicated in its pathophysiology: Multi-vessel epicardial coronary artery spasm, coronary microvascular impairment, direct catecholamine cardiotoxicity, and neurogenic stunned myocardium, although none of them can explain the wide spectrum of the disease [6,7]. A different hypothesis is that high levels of circulating epinephrine trigger to switch in intracellular signal trafficking, from Gs (stimulating) protein Gi (inhibitory) protein signaling through the beta-2 adrenoreceptor, producing a negatively inotropic effect that is greatest at the apical myocardium, in which the density of beta-adrenoreceptors is the highest [8-10].

The most frequent clinical symptoms of TC on admission are chest pain and dyspnea resembling acute myocardial infarction. Moreover, the ECG findings on admission often include ST elevation in precordial leads. Subsequent $T$ wave inversion and $\mathrm{Q}$ wave formation are also frequently found. However, coronary angiography, which is the best single tool to diagnose this condition, shows no flow-limiting lesions [2,3]. Although not a common finding, the right ventricle is also affected in some patients, being the most frequently affected segments: The apicolateral, the anterolateral, and the inferior segment, with all right ventricular wall-motion abnormalities showing improvement or disappearing in patients who underwent a follow-up cardiac magnetic resonance imaging (MRI) study. The left ventricular ejection fraction was lower in the cases

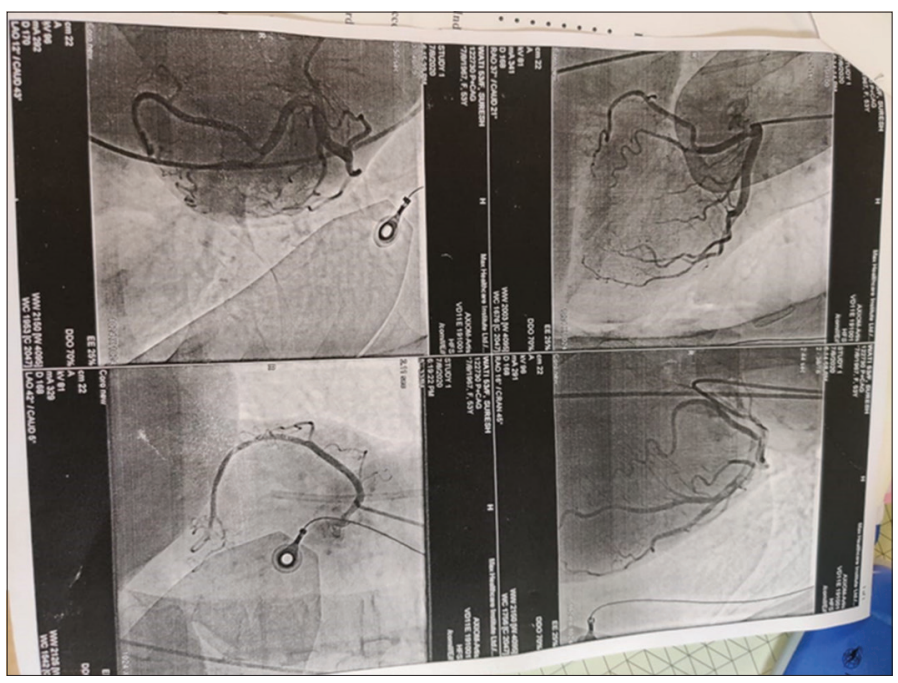

Figure 3: Coronary angiography

with the right ventricular affection, as well as pleural effusion was more common in patients with the right ventricular involvement [10].

Right and/or left ventricular thrombi and embolic events have been identified in some patients (predominantly by cardiac MRI). It has been reported that all-cause mortality during follow-up exceeds a matched general population, with most deaths occurring in the $1^{\text {st }}$ year. Nonfatal recurrent stress cardiomyopathy events have been also reported [8]. Emotional triggers have been found as common physical triggers. Moreover, in some patients, no evident trigger has been found. However, patients with TC have been found to have a higher prevalence of neurologic or psychiatric disorders than those with acute coronary syndrome [9].

There have been identified different morphological patterns of the left ventricular affection which includes apical and midventricular dysfunction, isolated mid-ventricular and basal dysfunction or isolated mid-ventricular dysfunction (apical sparing), isolated basal dysfunction, global hypokinesia, and other non-coronary distribution wall motion abnormalities [2]. Researchers at the Mayo Clinic proposed diagnostic criteria in 2004, which included (a) transient akinesis or dyskinesis of the left apical and mid-ventricular segments with regional wall-motion abnormalities extending beyond single epicardial vascular distribution, (b) an absence of obstructive coronary disease or angiographic evidence of acute plaque rupture, (c) new electrocardiographic abnormalities (either ST-segment elevation and/or T-wave inversion), and (d) absence of all of the following: recent significant head trauma, intracranial bleeding, pheochromocytoma, obstructive epicardial coronary artery disease, myocarditis, and hypertrophic cardiomyopathy [7].

However, certain evidence shows that coronary artery disease and TC can coexist, and takotsubo is particularly common in patients with pheochromocytoma or intracranial lesions [6]. Accordingly, $10 \%$ of patients with acute intracranial injury have 
acute ischemic electrocardiographic changes, the same clinical picture is seen in patients with elevated catecholamine levels secondary to pheochromocytomas [3].

Mayo clinic criteria were modified in 2008 , considering the (a) transient hypokinesis, akinesis, or dyskinesis in the left ventricular mid segments with or without apical involvement, (b) regional wall motion abnormalities that extend beyond a single epicardial vascular distribution, and frequently, but not always, a stressful trigger, the absence of obstructive coronary disease or angiographic evidence of acute plaque rupture; (c) new ECG abnormalities (ST-segment elevation and/or T-wave inversion) or modest elevation in cardiac troponin; and (d) the absence of pheochromocytoma and myocarditis. All four must be present to make the diagnosis $[5,6]$. In these modified criteria, the presence of intracranial lesions no longer excludes patients from a TC diagnosis. However, patients are still excluded from receiving TC diagnosis if pheochromocytoma or coronary artery disease is present [7].

The best imaging study to make the diagnosis of TC is cardiac catheterization; however, cardiac MRI allows complete anatomical information and may help to distinguish TC from other acute cardiac syndromes. Many recent studies have reported that the number of patients with mid-ventricular dysfunction is increased compared with the initial description of the disease. The atypical presentation with apical sparing and affection only in the basal segments is not rare [3-5].

There are no specific treatments for the left ventricular failure of TC because a cardiac function is normalized within a few weeks. The use of long-term adrenoceptor blocker therapy seems rational to prevent a recurrence, although its use in the acute phase of TC is still a matter of debate. Treatment with a combined alpha and beta-blocker seems rational, so we do not leave alpha-activity with an unopposed effect, favoring vasoconstriction. However, beta-blocking drugs have not always provided absolute protection against recurrent events $[2,3,8]$. Hypotension after the initiation of an angiotensin-converting enzyme inhibitor, angiotensin receptor blocker, or diuretics has been reported as a result of potentiation of a dynamic left ventricular outflow tract obstruction. A short duration of anticoagulation with warfarin may be considered for patients with a persistent, significant reduction in the left ventricle function to prevent thrombus formation and embolization [1,7]. The use of angiotensin-converting-enzyme inhibitors or angiotensinreceptor blockers has been associated with improved survival at 1 year [8]. Treatment with a catecholamine is usually not indicated since sympathetic activation is believed to be the basis of the pathogenesis of TC. The intra-aortic balloon pump is useful when shock occurs $[2,3]$.

The short-coming prognosis is well known, but there is a paucity of data from large and prospectively identified patient cohorts studied for extended periods of time following the initial event $[8,9]$. The prognosis of patients with TC is generally favorable $[1,10]$. However, such as pulmonary edema, intraventricular pressure gradients, acute mitral regurgitation, right ventricular involvement with pleural effusion, intraventricular thrombi resulting in a stroke or arterial embolism, atrial fibrillation, malignant ventricular arrhythmias, and cardiogenic shock and left ventricular free wall rupture can occur. Heart failure, with or without pulmonary edema, is the most common clinical complication. In-hospital mortality between 1 and $2 \%$ of patients $[3,11]$. The recurrence rate has been suggested to be in the range of $2-10 \%$ in the first few years after the presentation of TC. Recurrence rates may be lower if the adrenergic blockade is maintained, assuming the central role of catecholamines in the pathogenesis of the syndrome [2].

In the case of our patient, a takotsubo stress cardiomyopathy was diagnosed, after having found: apical and mid-ventricular hypokinesis with sparing of the basal segments; abnormal left ventricular wall motion affecting more than one epicardial coronary artery distribution, with normal coronary arteries on angiography, and an identified stressor factor; new ECG alterations accompanied by a modest increment of troponin I serum levels; and absence of myocarditis and pheochromocytoma as documented by cardiac MRI and urinary metanephrines, respectively. All of the four modified Mayo clinic criteria were satisfied.

\section{CONCLUSION}

TC is characterized as a transient left ventricular dysfunction with rapid recovery generally induced by a stressful emotional or physical event. The number of TC cases continues to increase due to the close resemblance of its presentation and clinical course to acute myocardial infarction; we state that TC should be included in one of the differential diagnoses for myocardial infarction. The occurrence of this disease is attributed to the large-scale production of catecholamines that cause myocardial hypokinesia. Although the treatment of TC remains controversial, the adrenergic blockade is suggested as a reasonable therapy based on the presumptive pathophysiology of TC.

\section{REFERENCES}

1. Brenton B, Solh T. Takotsubo cardiomyopathy: Review of broken heart syndrome. JAAPA 2020;33:24-9.

2. Bybee KA, Prasad A. Stress-related cardiomyopathy syndromes. Circulation 2008;118:397-409.

3. Lyon AR, Rees PS, Prasad S, Poole-Wilson PA, Harding SA. Stress (Takotsubo) cardiomyopathy--a novel pathophysiological hypothesis to explain catecholamine-induced acute myocardial stunning. Nat Clin Pract Cardiovasc Med 2008;5:22-9.

4. Akashi YJ, Goldstein DS, Barbaro G, Ueyama T. Takotsubo cardiomyopathy: A new form of acute, reversible heart failure. Circulation 2008;118:2754-62.

5. Gaspar J, Gómez Cruz RA. Tako-Tsubo syndrome (transient antero-apical dyskinesia): First case reported in Latin America and review of the literature. Arch Cardiol Mex 2004;74:205-14.

6. Camera MA, Dalia CG, Gómez FH. Takotsubo syndrome, report of two cases and review of the literature. Rev Asoc Mex Med Crit Ter Intensiva 2012;26:51-5.

7. Redfors B, Shao Y, Lyon AR, Omerovic E. Diagnostic criteria for Takotsubo syndrome: A call for consensus. Int J Cardiol 2014;176:274-6. 
8. Sharkey SW, Windenburg DC, Lesser JR, Maron MS, Hauser RG, Lesser JN, et al. Natural history and expansive clinical profile of stress (Tako-Tsubo) cardiomyopathy. J Am Coll Cardiol 2010;55:333-41.

9. Templin C, Ghadri JR, Diekmann J, Napp LC, Bataiosu DR, Jaguszewski M, et al. Clinical features and outcomes of Takotsubo (stress) cardiomyopathy. N Engl J Med 2015;373:929-38.

10. Haghi D, Athanasiadis A, Papavassiliu T, Suselbeck T, Fluechter S, Mahrholdt $\mathrm{H}$, et al. Right ventricular involvement in Takotsubo cardiomyopathy. Eur Heart J 2006;27:2433-9.

11. Bossone E, Lyon A, Citro R, Athanasiadis A, Meimoun P, Parodi G, et al.
Takotsubo cardiomyopathy: An integrated multi-imaging approach. Eur Heart J Cardiovasc Imaging 2014;15:366-77.

Funding: None; Conflicts of Interest: None Stated.

How to cite this article: Sharma K, Nair D, Monga J, Sehgal P. A Case Report of Takotsubo Cardiomyopathy. Indian J Case Reports. 2020;6(10):583-586. 